\title{
Chenodeoxycholate Is a Potent Inducer of the Permeability Transition Pore in Rat Liver Mitochondria
}

\author{
Anabela P. Rolo, ${ }^{1}$ Paulo J. Oliveira, ${ }^{1}$ António J. M. Moreno, ${ }^{1}$ and \\ Carlos M. Palmeira ${ }^{1,2}$
}

Received November 17, 2000

Several reports support the concept that bile acids may be cytotoxic during cholestatic disease process by causing mitochondrial dysfunction. Here we report additional data and findings aimed at a better understanding of the involvement of the permeability transition pore (PTP) opening in bile acids toxicity. The mitochondrial PTP is implicated as a mediator of cell injury and death in many situations. In the presence of calcium and phosphate, chenodeoxycholic acid (CDCA) induced a permeability transition in freshly isolated rat liver mitochondria, characterized by membrane depolarization, release of matrix calcium, and osmotic swelling. All these events were blocked by cyclosporine A (CyA) and the calcium uniporter inhibitor ruthenium red (RR). The results suggest that CDCA increases the sensitivity of isolated mitochondria in vitro to the calcium-dependent induction of the PTP.

KEY WORDS: Mitochondria; permeability; chenodeoxycholic acid; calcium; membrane potential.

\begin{abstract}
ABBREVIATIONS: $\Delta \Psi$, mitochondrial membrane potential; $\mathrm{TPP}^{+}$, tetraphenylphosphonium cation; PTP, permeability transition pore; RR, ruthenium red; CyA, cyclosporine A; CDCA, chenodeoxycholic acid.
\end{abstract}

\section{INTRODUCTION}

Liver diseases such as cholestasis are a significant clinical problem, but the cellular mechanisms disturbed during these pathophysiological processes are still controversial. Retention of toxic, hydrophobic bile acids occurs during cholestasis [1]. The accumulation of toxic bile acids results in chemical damage to hepatocytes and is thought to be responsible, at least in part, for liver injury during cholestasis [2].

The tendency to cause cytotoxicity has been correlated with bile acids hydrophobicity $[3,4]$. Chenodeoxycholic acid (CDCA), a primary bile acid most implicated in cholestatic liver injury, is known to be directly cytotoxic for hepatocytes

${ }^{1}$ Center for Neurosciences and Cell Biology of Coimbra, Department of Zoology, University of Coimbra, 3004-517 Coimbra, Portugal.

${ }^{2}$ To whom correspondence should be addressed. Fax: (351) (239) 826798; E-mail: palmeira@ci.uc.pt 
[5]. Hepatic tissue concentrations of CDCA increase 20-fold during cholestasis and are greater than those of other toxic bile acids [6].

Impairment of mitochondrial function seems to be a central biochemical pathway of cell dysfunction during the cholestatic disease process. ATP depletion observed after addition of glycochenodeoxycholate, even in the absence of a glycolytic substrate, suggests disturbing of mitochondrial function [7]. Long-term cholestasis caused by bile duct ligation is known to lead to impaired hepatic mitochondrial function in the rat [8] and enlarged, swollen mitochondria are observed in histopathologic sections obtained from this model of extrahepatic cholestasis [9]. Additionally, bile acid-induced decrease on state 3 respiration in isolated rat liver mitochondria $[10,11]$ and in digitonin-permeabilized cells [7], has been reported. Since mitochondria are provided with a variety of bioenergetic functions mandatory for the regulation of intracellular aerobic energy production and electrolyte homeostasis, impairment of mitochondrial function by bile acids may have drastic consequences on cellular function through the perturbation of the bioenergetic charge and balance of the cell.

Mitochondria are also involved in cell demise through opening of the mitochondrial permeability transition pore (PTP). PTP induction is implicated in the mechanism of cell killing by a number of agents including heavy metals, pesticides, and selected pharmaceuticals $[12,13]$. The transition is characterized by a calciumdependent increase in the non-specific permeability of the inner mitochondrial membrane accompanied by the depolarization of membrane potential and mitochondrial swelling. Induction of the transition requires adequate matrix calcium and phosphate and is inhibited by extramitochondrial calcium and acidification of the mitochondrial matrix [14-16]. Cyclosporine A (CyA) is a potent inhibitor of the permeability transition [17].

Recent data have reported the induction of the PTP as a mechanism of cytotoxicity of bile acids $[11,18,19]$. The mechanism by which bile acids promote the onset of PTP remains unclear. The present study attempted to gain further insight into CDCA effects on the mitochondrial permeability transition, in order to better elucidate possible mechanisms of action.

\section{MATERIALS AND METHODS}

\section{Chemicals}

CDCA, used as ethanolic solution was purchased from Sigma Chemical Co. (St. Louis, MO). Cyclosporine A was a generous gift from Novartis Pharma AG (East Hanover, NJ). All other chemicals were of the highest grade of purity commercially available.

\section{Isolation of Mitochondria}

Mitochondria were isolated from liver of male Wistar rats by conventional methods [20] with slight modifications. Homogenization medium contained $250 \mathrm{mM}$ sucrose, $10 \mathrm{mM}$ Hepes ( $\mathrm{pH} 7.4), 0.5 \mathrm{mM}$ EGTA, and $0.1 \%$ fat-free bovine serum 
albumin. EGTA and bovine serum albumin were omitted from the final washing medium, adjusted at $\mathrm{pH}$ 7.4. The mitochondrial pellet was washed twice, suspended in the washing medium, and immediately used. Protein content was determined by the biuret method [21] calibrated with bovine serum albumin.

\section{Membrane Potential $(\Delta \Psi)$ Measurements}

The mitochondrial transmembrane potential was estimated using an ion-selective electrode to measure the distribution of tetraphenylphosphonium $\left(\mathrm{TPP}^{+}\right)$ according to previously established methods [22,23]. The reference electrode was $\mathrm{Ag} / \mathrm{AgCl}_{2}$. The incubation medium contained $200 \mathrm{mM}$ sucrose, $10 \mathrm{mM}$ Tris-Mops (pH 7.4), $1 \mathrm{mM} \mathrm{KH}_{2} \mathrm{PO}_{4}$, and $10 \mu \mathrm{M}$ EGTA supplemented with $2 \mu \mathrm{M}$ rotenone and $0.5 \mu \mathrm{g} / \mathrm{ml}$ oligomycin. Mitochondria $(0.5 \mathrm{mg})$ were suspended with constant stirring, at $25^{\circ} \mathrm{C}$, in $1 \mathrm{ml}$ of the standard incubation medium supplemented with $3 \mu \mathrm{M} \mathrm{TPP}^{+}$ and were energized by adding succinate to a final concentration of $5 \mathrm{mM}$. The distribution of $\mathrm{TPP}^{+}$was allowed to reach a new equilibrium (ca. $2 \mathrm{~min}$ ) before making any further addition. The electrode was calibrated with $\mathrm{TPP}^{+}$assuming Nerstian distribution of the ion across the synthetic membrane. A matrix volume of $1.1 \mu \mathrm{l} /$ mg protein was assumed.

\section{Measurement of the Mitochondrial Permeability Transition}

Mitochondrial swelling was estimated by changes in light-scattering as monitored spectrophotometrically at $540 \mathrm{~nm}$ [24]. The reaction was stirred continuously and the temperature maintained at $25^{\circ} \mathrm{C}$. The experiments were started by the addition of $1 \mathrm{mg}$ of mitochondria to a final volume of $2 \mathrm{ml}$ of the standard incubation medium, supplemented with $2 \mu \mathrm{M}$ rotenone, $0.5 \mu \mathrm{g} / \mathrm{ml}$ oligomycin, and $5 \mathrm{mM}$ succinate. Calcium $\left(20 \mu \mathrm{M} \mathrm{CaCl}_{2}\right)$ was added prior to all other compounds.

\section{Measurement of Mitochondrial Calcium Fluxes}

The accumulation and release of calcium by isolated rat liver mitochondria was determined using a calcium-sensitive fluorescence dye, calcium green-5N [25]. The reactions were carried out at $25^{\circ} \mathrm{C}$, in $2 \mathrm{ml}$ of the standard incubation medium, supplemented with $2 \mu \mathrm{M}$ rotenone, $0.5 \mu \mathrm{g} / \mathrm{ml}$ oligomycin, and $0.1 \mu \mathrm{M}$ calcium green$5 \mathrm{~N}$. Mitochondria $(0.4 \mathrm{mg})$ were suspended with constant stirring. Fluorescence (ex., $505 \mathrm{~nm}$; em., $531 \mathrm{~nm}$ ) was monitored continuously for an additional 1.5 min prior to adding calcium $\left(\mathrm{CaCl}_{2}\right)$ to a final concentration of $5 \mu \mathrm{M}$. Fluorescence was monitored continuously for an additional $3 \mathrm{~min}$, at which time $8 \mathrm{mM}$ succinate was added to the suspension. At each experiment, fluorescence was monitored for an additional 10 min before stopping the reaction with excess EGTA. Calcium fluxes are expressed as relative fluorescence units (RFU). At the concentration examined, CDCA did not interfere with the experimental assay. 


\section{RESULTS}

Figure 1 illustrates the effect of CDCA on membrane potential $(\Delta \Psi)$, in hepatic mitochondria energized with succinate. Upon addition of calcium $(15 \mu \mathrm{M})$ to mitochondria, a transient depolarization of membrane potential occurred, followed by repolarization to the initial resting membrane potential. Subsequent addition of $50 \mu \mathrm{M}$ CDCA resulted in an immediate decrease of membrane potential, which was not prevented by CyA. Calcium-loaded mitochondria, in the presence of CDCA, were incapable of sustaining membrane potential and underwent a gradual depolarization over time. This is the result of PTP induction and the associated loss of regulation of transmembrane ionic gradients. Pretreatment with both $\mathrm{CyA}$ and ruthenium red (RR) prevented this CDCA-induced dramatic depolarization (Fig. 1).

Mitochondria possess a finite capacity for accumulating calcium before undergoing the calcium-dependent PTP. Figure 2 illustrates the results achieved when we

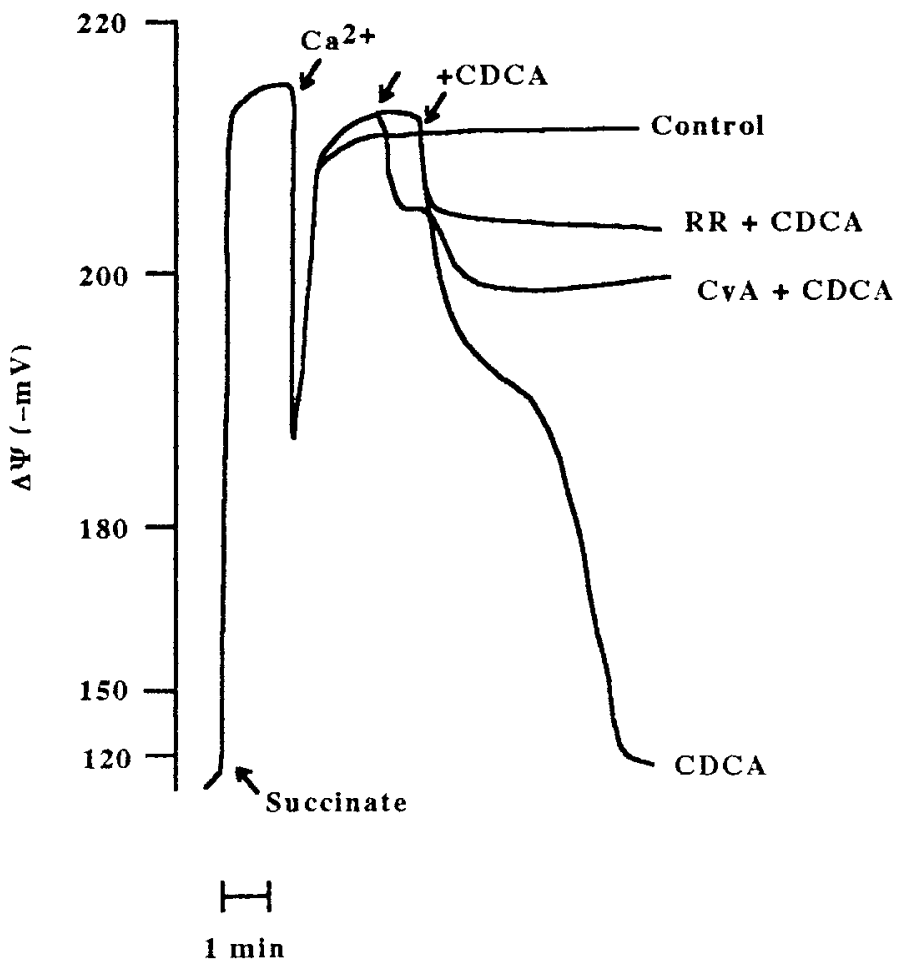

Fig. 1. Effect of chenodeoxycholic acid (CDCA) on mitochondrial transmembrane potential $(\Delta \Psi)$. Mitochondria $(0.5 \mathrm{mg})$ were incubated in $1 \mathrm{ml}$ of the standard incubation medium supplemented with $2 \mu \mathrm{M}$ rotenone, $0.5 \mu \mathrm{g} / \mathrm{ml}$ oligomycin, and $3 \mu \mathrm{M} \mathrm{TPP}^{+}$and energized with $5 \mathrm{mM}$ succinate. Calcium was added at a final concentration of $15 \mu \mathrm{M}$. Where indicated (first arrow), $1 \mu \mathrm{M}$ CyA or $1 \mu \mathrm{M}$ ruthenium red (RR) were included in the reaction medium prior to addition of $50 \mu \mathrm{M}$ CDCA. The traces are typical of several independent experiments with four different mitochondrial preparations. 
follow mitochondrial swelling as the indicator for induction of the PTP by CDCA. As we reported previously [11], calcium-loaded, succinate-energized rat liver mitochondria underwent a permeability transition, as reflected by the decreased absorbance at $540 \mathrm{~nm}$, upon addition of CDCA. In all cases, the induction of mitochondrial swelling by CDCA required calcium and was inhibited completely by prior addition of CyA (Fig. 2). Furthermore, PTP induction by CDCA was also prevented upon preincubation of calcium-loaded mitochondria with $1 \mu \mathrm{M}$ RR (Fig. 2). No swelling was observed in control mitochondria following calcium addition.

Mitochondrial calcium homeostasis was also adversely affected by CDCA. Unlike control mitochondria, which accumulated all of the added calcium and retained it for the entire period monitored, after addition of CDCA, mitochondria released all the accumulated calcium (Fig. 3).

The protective effect of CyA against CDCA-induced mitochondrial calcium release was assessed to evaluate the involvement of PTP induction in this phenomenon. Indeed, including CyA prior to CDCA, completely prevented mitochondrial calcium release. Similar results were achieved by adding RR at the same time as CyA (data not shown), which is what would be predicted from the results of membrane potential and swelling experiments.

Furthermore, adding RR during the course of CDCA-induced calcium release prevented the release of all the accumulated calcium (Fig. 3). It seems likely that RR inhibited the net reaccumulation of released calcium from mitochondria, implicating the electrophoretic uniport in the reuptake of the released calcium.

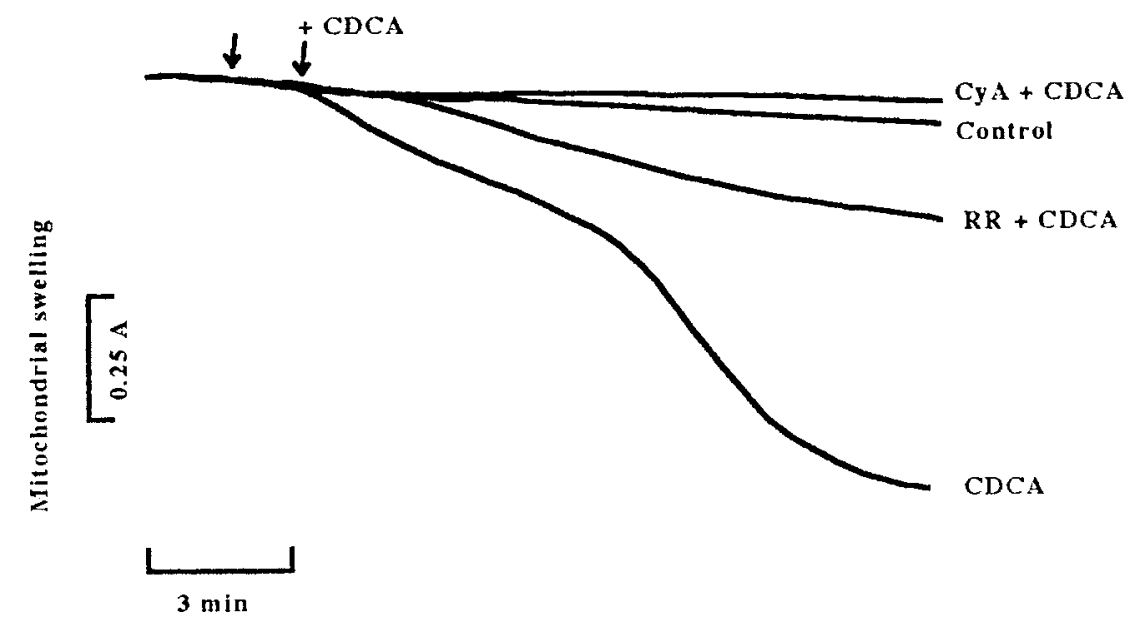

Fig. 2. Effect of chenodeoxycholic acid (CDCA) on PTP induction. The incubation medium (final volume $2 \mathrm{ml}$ at $25^{\circ} \mathrm{C}$ ) contained $200 \mathrm{mM}$ sucrose, $10 \mathrm{mM}$ Tris-Mops (pH 7.4), $1 \mathrm{mM}$ $\mathrm{KH}_{2} \mathrm{PO}_{4}$, and $10 \mu \mathrm{M}$ EGTA supplemented with $2 \mu \mathrm{M}$ rotenone, $0.5 \mu \mathrm{g} / \mathrm{ml}$ oligomycin, and $5 \mathrm{mM}$ succinate. The experiments were started by the addition of $1 \mathrm{mg}$ of mitochondria (not shown). Calcium $(20 \mu \mathrm{M})$ was added prior all other compounds. Where indicated (first arrow), $1 \mu \mathrm{M}$ CyA, or $1 \mu \mathrm{M}$ ruthenium red (RR) were included in the reaction medium prior to $50 \mu \mathrm{M}$ CDCA. The traces are typical of several independent experiments with four different mitochondrial preparations. 


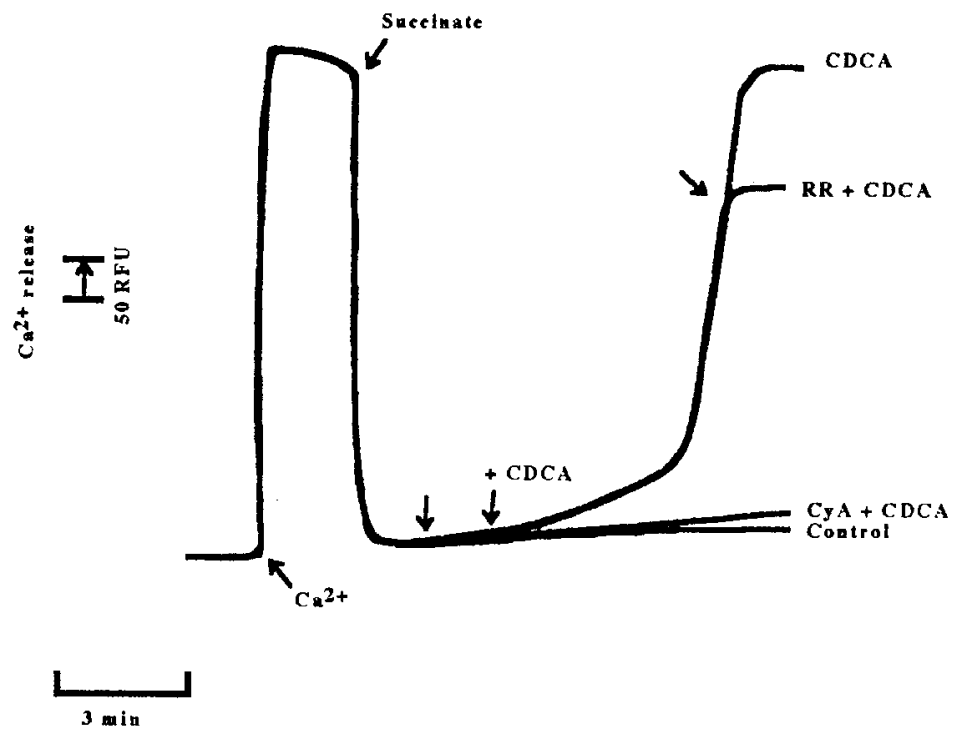

Fig. 3. Chenodeoxycholic acid (CDCA)-induced mitochondrial calcium release. Mitochondria $(0.4 \mathrm{mg})$ were incubated in $2 \mathrm{ml}$ of the standard incubation medium prior to calcium $(5 \mu \mathrm{M})$. Calcium fluxes, expressed as relative fluorescence units (RFU), was recorded for an additional $13 \mathrm{~min}$. When indicated, CyA $(0.4 \mu \mathrm{M})$ or ruthenium red (RR; $0.4 \mu \mathrm{M})$ was added prior or after, respectively, to CDCA $(20 \mu \mathrm{M})$. Excess EGTA was added at the end of the experiment. The traces are typical of several independent experiments with three different mitochondrial preparations. An upward pen deflection is indicative of calcium release.

\section{DISCUSSION}

In a previous report, we indicated that mitochondria were likely the subcellular target for bile acids hepatotoxicity [11]. It was suggested that a perturbation of mitochondrial bioenergetics was involved in the cytotoxic action of these compounds. The present study provides new data useful to understand the mechanisms whereby bile acids elicit liver injury. Data obtained with isolated rat liver mitochondria suggest that $\mathrm{CDCA}$, a primary bile acid most implicated in cholestatic liver injury, may alter the regulation of the cyclosporine A-sensitive permeability transition pore.

PTP opening is widely accepted to be an early event in pathophysiology of cell death [26]. It is characterized by mitochondrial membrane depolarization, mitochondrial calcium release, and an increase in non-specific permeability of the inner membrane to low-molecular-weight solutes, leading to mitochondrial swelling and inhibition of oxidative phosphorylation. The PTP is suggested to be composed of a complex of distinct proteins, which accounts for the coordinated regulation of the pore by assorted factors, including several chemically diverse inducing agents [27]. Some key features of the PTP are that it has a strict requirement for matrix calcium and is selectively inhibited by CyA [17]. 
The detection of membrane potential loss and osmotic swelling, after adding CDCA to calcium-loaded isolated mitochondria, suggests the sensitization of mitochondria to PTP opening, probably at the level of calcium-binding sites on the matrix side of the membrane. Associated with induction of mitochondrial swelling by CDCA, was a pronounced loss of matrix calcium. The sensitivity of PTP activation to CyA was used to confirm its involvement in the observed disruption of mitochondrial calcium homeostasis. Complete inhibition of both mitochondrial swelling and matrix calcium release by CyA, suggests a selective effect of CDCA on this specific channel. The fact that $\mathrm{CyA}$ completely prevented the reduction in maximum calcium accumulation by mitochondria, in the presence of CDCA, indicates that the lower capacity to accumulate calcium represents a stimulation of calcium release rather than inhibition of calcium uptake. This is further evidenced by the success of preventing the observed CDCA effects by using ruthenium red.

Results provide evidence for the opening of this pore within a subpopulation of hepatic mitochondria, upon adding CDCA. At the level of a single mitochondrion the PTP opening is an all-or-nothing event. The kinetics observed in light-scattering experiments, i.e., on a time scale of minutes, reflect therefore the propagation of the transition through the population of mitochondria [27]. PTP-induced loss of transmembrane potential will also lead to the efflux of calcium which the mitochondria had originally been loaded. The released calcium may be taken up by still-coupled mitochondria, which would also undergo CDCA-induced PTP opening. When the uptake uniport was inhibited with RR, calcium was not reaccumulated. The ability to reaccumulate the released calcium indicates that the mitochondria were capable of establishing a membrane potential sufficient to support the electrophoretic calcium uptake uniport [28]. The preventative action of RR therefore reflects differing sensitivities among subpopulations of mitochondria.

In summary, our data provide further evidence for the permeability transition of the membrane of individual mitochondria caused by CDCA, in vitro. We conclude that this may be an important step within the cascade of events which induce breakdown of mitochondria and cellular function, involved in the cholestatic disease process. The precise mechanism by which CDCA interferes with regulation of the pore has yet to be defined.

\section{ACKNOWLEDGMENTS}

A. P. Rolo and P. J. Oliveira are the recipients of grant Praxis XXI/BD/21454/ 99 and PRAXIS XXI/BD/21494/99, respectively, from Fundação para a Ciência e a Tecnologia (FCT), Lisbon, Portugal.

\section{REFERENCES}

1. Greim, H. et al. (1972) Gastroenterology 63:846-850.

2. Kaplan, M. (1994) N. Engl. J. Med. 330:1368-1387.

3. Armstrong, M. J. and Carey, M. C. (1982) J. Lipid. Res. 23:70-80.

4. Attili, A. F., Angelico, M., Cantafora, A., Alvaro, D., and Capocaccia, L. (1986) Med. Hypoth. 19:57-69.

5. Miyazaki, K., Nakayama, F., and Koga, A. (1984) Dig. Dis. Sci. 12:1123-1130. 
6. Greim, H., Czygan, P., Schaffner, F., and Popper, H. (1973) Biochem. Med. 8:280-286.

7. Spivey, J. R., Bronk, S.F., and Gores, G. (1993) J. Clin. Invest. 92:17-24.

8. Krähenbühl, S., Stucki, J., and Reichen, J. (1992) Hepatology 15:1160-1166.

9. Schaffner, F. et al. (1971) Gastroenterology 60:888-897.

10. Krähenbühl, S., Talos, C., Fischer, S., and Reichen, J. (1994) Hepatology 19:471-479.

11. Rolo, A. P., Oliveira, P. J., Moreno, A. J. M., and Palmeira, C. M. (2000) Toxicol. Sci. 57:177-185.

12. Bernardi, P., Veronese, P., and Petronilli, V. (1993) J. Biol. Chem. 268:1005-1010.

13. Gunter, T. E., Gunter, K. K., Sheu, S.-S., and Gavin, C. E. (1994) Am. J. Physiol. 267:C313-C339.

14. Bernardi, P., Vassanelli, S., Veronese, P., Colonna, R., Szabo, I., and Zoratti, M. (1992) J. Biol. Chem. 267:2934-2939.

15. Bernardi, P., Broekemeier, K. M., and Pfeiffer, D. R. (1994) J. Bioenerg. Biomembr. 26:509-517.

16. Petronilli, V., Cola, C., and Bernardi, P. (1993) J. Biol. Chem. 268:1011-1016.

17. Broekemeier, K. M., Dempsey, M. E., and Pfeiffer, D. R. (1989) J. Biol. Chem. 264:7826-7830.

18. Botla, R., Spivey, J. R., Aguilar, H., Bronk, S. F., and Gores, G. J. (1995) J. Pharmacol. Exp. Ther. 272:930-938.

19. Gores, G. J., Miyoshi, H., Botla, R., Aguilar, H. I., and Bronk, S. F. (1998) Biochim. Biophys. Acta 1366: $167-175$.

20. Gazotti, P., Malmstron, K., and Crompton, M. (1979) In: Membrane Biochemistry. A Laboratory Manual on Transport and Bioenergetics (E. Carafoli and G. Semenza, eds.), Springer-Verlag, New York, pp. 62-69.

21. Gornall, A. G., Bardawill, C. J., and David, M. M. (1949) J. Biol. Chem. 177:751-766.

22. Kamo, N., Muratsugu, M., Hongoh, R., and Kobatake, V. (1979) J. Membr. Biol. 49:105-121.

23. Palmeira, C. M., Moreno, A. J., and Madeira, V. M. C. (1994) Toxicol. Appl. Pharmacol. 127:338347.

24. Palmeira, C. M. and Wallace, K. B. (1997) Toxicol. Appl. Pharmacol. 143:338-347.

25. Rajdev, S. and Reynolds, I. J. (1993) Neurosci. Lett. 162:149-152.

26. Zamzami, N., Hirsch, T., Dallaporta, B., Petit, P. X., and Kroemer, G. (1997) J. Bioenerg. Biomembr. 29:185-193.

27. Zoratti, M. and Szabo, I. (1995) Biochim. Biophys. Acta 1241:139-176.

28. Gunter, T. E. and Pfeiffer, D. R. (1990) Am. J. Physiol. 258:C755-C786. 